\title{
Biodegradable collagen matrix implant versus mitomycin-C in trabeculectomy: five-year follow-up
}

\author{
Salvatore Cillino ${ }^{1 *}$, Alessandra Casuccio ${ }^{2}$, Francesco Di Pace $^{1}$, Carlo Cagini $^{3}$, Lucia Lee Ferraro ${ }^{1}$
} and Giovanni Cillino ${ }^{1}$

\begin{abstract}
Background: Clinical studies comparing trabeculectomy augmented with Ologen implant (OLO) versus trabeculectomy plus mitomycin-C (MMC) show contradictory results. To obtain long-term data, we report an extended 5-year follow-up trial evaluating the safety and efficacy of OLO as adjuvant compared to low-dosage MMC in trabeculectomy.
\end{abstract}

Methods: Forty glaucoma patients (40 eyes) assigned to trabeculectomy with MMC or Ologen. Primary outcome: target IOP at $\leq 21, \leq 17$ and $\leq 15 \mathrm{mmHg}$; complete and qualified success endpoint rates. Secondary outcomes: visual acuity (VA), mean deviation (MD), bleb evaluation, according to Moorfields Bleb Grading System (MBGS); spectral domain OCT (SD-OCT) bleb examination; number of glaucoma medications; frequency of postoperative complications.

Results: The mean preoperative IOP was $26.7( \pm 5.2)$ in MMC and 27.3( \pm 6.0$)$ in OLO eyes. Mean 60-month percentage reduction in IOP was significant in both groups [40.9 $( \pm 14.2)$ and $42.1( \pm 13.3) P=0.01]$, with an endpoint value of $15.2( \pm 3.2)$ and $15.8( \pm 2.3) \mathrm{mmHg}$ in $\mathrm{MMC}$ and $\mathrm{OLO}$, respectively. Complete success rates at $\leq 21 \mathrm{mmHg}$ target IOP were $65 \%$ and $70 \%$, at $\leq 17 \mathrm{~mm} \mathrm{Hg} 60 \%$ and $55 \%$, and at the $\leq 15 \mathrm{~mm} \mathrm{Hg}$ target IOP $35 \%$ and $45 \%$ in MMC and OLO, respectively.

The Kaplan-Meier curves did not differ both for complete and qualified success at any target IOP, with no significant endpoint intergroup difference at $\leq 15 \mathrm{~mm} \mathrm{Hg}$ (log-rank $P=0.595$ ). The intergroup MBGS scores differed due to reduced central and peripheral vascularity in MMC group $(P=0.027 ; P=0.041)$.

SD-OCT analysis denied differences in bleb height between MMC vs OLO $(140.5 \pm 20.3 \mu$ vs $129.2 \pm 19.3 \mu$ respectively; $P=0.079$ ).

Mean antiglaucoma medications were significantly reduced $(P<0.0005)$ from $2.5( \pm 0.3)$ to $1.2( \pm 0.4)$ in MMC and from $2.6( \pm 0.2)$ to $1.4( \pm 0.3)$ in OLO group, with no intergroup differences $(P=0.08)$.

Six (30 \%) cystic thin avascular blebs without oozing were recorded in the MMC group and $2(10 \%)$ in the OLO group, without intergroup difference $(P=0.235)$.

Conclusions: Our extended follow-up results confirm that Ologen implant yields efficacy and long-term success rates quite similar to MMC, with at least equivalent safety.

Keywords: Mitomycin-C, Ologen, Trabeculectomy, Extended 5-yrs follow-up

\footnotetext{
* Correspondence: salvatore.cillino@unipa.it

${ }^{1}$ Department of Experimental Biomedicine and Clinical Neuroscience,

Ophthalmology Section, University of Palermo (Italy), via Liborio Giuffrè, 13,

90127 Palermo, Italy

Full list of author information is available at the end of the article
} 


\section{Background}

Trabeculectomy with mitomycin-C (MMC) today is still regarded as the gold-standard in glaucoma surgery. Yet, in many studies MMC-related complications such as prolonged wound leaks, hypotony with choroidal effusions and maculopathy, thin avascular blebs, and/or bleb leaks with late infection are frequently reported [1-9].

A biodegradable collagen-glycosaminoglycan copolymer matrix implant (Ologen ${ }^{\circ}$ ) has been proposed as an alternative adjuvant, used as a spacer to mechanically separate the sub conjunctival and episcleral tissues to preventing fibrosis, and also helps in reorganizing the subconjunctival scar formation.

In fact, it should induce fibroblasts and myofibroblasts to grow randomly into its porous structure and secrete a loose connectival matrix, reducing the scarring degree. The implant is recommended to be placed subconjunctivally over the scleral flap posteriorly and possibly a small portion covering the scleral flap, else the ologen disc would act as a mechanical tamponade and prevent fluid outflow from the sub scleral space.

In 2010, a medium-term RCT did not show any intraocular pressure-lowering advantage of the Ologenaugmented trabeculectomy vs trabeculectomy alone, with a higher yet not significant incidence of complications with the collagen implant [10]. In the same year another randomized study of MMC-augmented trabeculectomy vs trabeculectomy using Ologen showed a lower complete success rate but a lower bleb-associated complication rate in Ologen group [11].

In 2011, we published the results of a 24-month, randomized prospective clinical trial on Ologen implant vs MMC in trabeculectomy [12]. The intraocular pressure (IOP) reduction was significant at endpoint in all groups $(P=0.01)$. The rates and Kaplan-Meier curves did not differ for both complete and qualified success at any target IOP. The bleb height in the Ologen-treated group was higher than in the MMC one $(P<0.05)$. No adverse reaction to Ologen was noted.

In the past three years, a number of clinical studies have compared the efficacy and tolerability of trabeculectomy augmented with Ologen versus trabeculectomy plus MMC, with somehow contradictory results [13-20].

To obtain more data on the long-term IOP lowering effect of the Ologen implant compared to MMC as adjuvant in trabeculectomy, we extended to five years the follow-up on the same cohort of subjects of our abovesaid study. The parameters measured included IOP, bleb morphology, and frequency of complications.

\section{Methods}

This study is an extended, 60-month follow-up data. The protocol of the randomized study had been approved by the Ethical Committee of the University
Hospital of Palermo (Italy) on December 2007 and registered under the number 08/07 (12 September 2007).We reviewed the records of 40 patients who had been randomly assigned to undergo a trabeculectomy with MMC (MMC group) or a trabeculectomy with Ologen implant (OLO group) for primary open-angle (POAG) or pseudoexfoliative glaucoma (PEXG) at the Department of Ophthalmology of the University of Palermo. The data used to generate the 5-year life table analysis were collected over the interval between enrollment in the study and 60 months following surgery.

In the previous prospective randomized clinical trial [12] a sample size of 40 patients (20 eyes in each group) had been chosen to achieve a power of $90 \%$ for detecting a $3-\mathrm{mmHg}$ difference in IOP between treatment procedures, assuming a standard deviation of three $\mathrm{mmHg}$ and a two-sided $\alpha$ error of $5 \%$.

In accord with tenets of Declaration of Helsinki a written informed consent has been obtained from all patients, also covering approval to publish images. We screened for uncontrolled glaucoma 65 consecutive Caucasian patients at the Glaucoma Center of the Department of Ophthalmology between January and December 2008. Sealed envelope technique from surgical chart number was used to ensure randomization just before surgery. Skilled ophthalmologists and optometrists masked to randomization collected the clinical data and the outcomes. Inclusion criteria were age 18 or older, diagnosis of POAG or PEXG with mild to moderate visual field damage [21], IOP above $21 \mathrm{~mm} \mathrm{Hg}$ or visual field deterioration on maximumtolerated medical therapy.

Exclusion criteria were advanced glaucoma or split fixation on visual field, normal-tension glaucoma, use of medications for acute or chronic disease that could affect the outcomes (eg, immunodeficiency, connective tissue disease, and diabetes), clinically significant cataract, previous ocular trauma or surgery. Included preoperative data consisted of age, gender, type of glaucoma, type and number of antiglaucoma medications; the ophthalmic examination included Goldmann applanation tonometry, biomicroscopy; Snellen visual acuity and computerized Humphrey visual field testing (Humphrey Visual Field Analyzer; HFA; Carl Zeiss Meditec. Inc.).

The primary outcome was IOP evaluated at three different IOP target levels: $\leq 21, \leq 17$, and $\leq 15 \mathrm{mmHg}$. Complete and qualified success were defined as usual, i.e. without medications in the first case and regardless of medications in the second one. Secondary outcome measures included visual acuity (VA), mean deviation (MD) change by Humphrey 24-2 full threshold testing, bleb evaluation according to Moorfields Bleb Grading System (MBGS), and bleb SD-OCT analysis. Number of glaucoma medications and frequency of postoperative 
adjunctive procedures and complications were also evaluated.

\section{Surgical technique and follow-up}

The technique has been described in detail in the previous study [12]. All operations, carried out by one experienced surgeon (SC), included a superior fornix-based conjunctival/tenons flap, with a rectangular scleral flap at the 12-o'clock position, fashioned according to the 'Moorfields Safer Surgery System' procedure [22, 23]. When MMC (Kyowa S.r.l., Milan, Italy) was the randomized adjunctive therapy, Weck-cell sponge pieces soaked with $0.2 \mathrm{mg} / \mathrm{ml} \mathrm{MMC}$ were simultaneously placed under the dissected conjunctiva/tenon surrounding the scleral flap [22, 23], and on the scleral bed, underneath the scleral flap, for a total time of $2 \mathrm{~min}$ [24]. After thorough irrigation, trabeculectomy was performed with a Crozafon-De Laage punch. A peripheral iridectomy was performed, the anterior chamber was filled with viscoelastic device, and the scleral flap was closed with two minimally tensed 10-0 nylon sutures -one at each corner- in MMC cases and with one long and loose stitch in OLO cases. A cylindrical Ologen implant (model number 830601, Aeon Astron Europe BV), $2.0 \pm 0.3 \mathrm{~mm}$ in height $\mathrm{x} 6.0 \pm 0.5 \mathrm{~mm}$ in diameter was then centered over the top of scleral flap and underneath the conjunctiva in the latter leaving the limbal end of the stitch partially uncovered. The implants used in this cohort of patients consisted of porcine based lyophilized crosslinked type I atelocollagen ( $\geq 90 \%)$ and glycosaminoglycans $(\leq 10 \%)$. Degradation time of this type was around 180 days. A newer version with exactly the same composition but with a bit shorter degradation time is now available.

The conjunctival flap was secured to the limbus with a tight 10-0 nylon running suture with buried knots.

Postoperatively, all eyes were treated with tapering doses of topical tobramycin $0.3 \%$ and dexamethasone drops $0.1 \%$ for 2 months. The 'intensified postoperative care' (IPC) protocol [25] was followed in all cases and included more frequent topical steroid administration if corkscrew bleb vessels were present. Instillation of $1 \%$ atropine drops during the first few days was based on the hypotony degree. Adjunctive procedures included the Carlo Traverso maneuver [26], laser suture lysis or bleb needling of encapsulated blebs (without antimetabolites).

If postoperative IOP measurements were $>21 \mathrm{mmHg}$ after topical steroid withdrawal, IOP-lowering medication was added.

When, during the extended follow-up, a patient developed a clinically significant cataract in the operated eye, a standard sutureless cataract surgery was performed through a temporal $2.5-\mathrm{mm}$ near-clear corneal tunnel incision with a precalibrated knife (Clearcut, Alcon Italia
S.P.A., Milan, Italy). Phacoemulsification was performed with the Alcon Infiniti Vision System (Alcon Italia S.P.A.), using the Ozil torsional handpiece in the majority of cases, avoiding any contact with the bleb area. The implantation of acrylic hydrophobic foldable intraocular lenses (IOLs) was performed using an Unfolder Emerald injector system (AMO Italy, Rome, Italy) or a Monarch II injector (Alcon Italia S.P.A.). The surgical wound was closed by stromal hydration. All patients received topical ofloxacin (Exocin, Allergan SpA, Rome, Italy) for 3 days preoperatively and tobramycin and dexamethasone ophthalmic suspension (Tobradex, Alcon Italia S.P.A.) for one week postoperatively, followed by nepafenac ophthalmic suspension (Nevanac, Alcon Italia S.P.A.) or bromfenac ophthalmic solution (Yellox, Bausch Lomb, Italia), for 3 weeks. In case of topical antiglaucomatous therapy use, this was continued even throughout the cataract surgery period. Even in cataract surgery cases, if postoperative IOP measurements were $>21 \mathrm{mmHg}$ after topical steroid withdrawal, IOP-lowering medication was added.

Expanded follow-up implied six-monthly examinations till the $60^{\text {th }}$ month. All patients were thoroughly informed about the importance of a periodical examination, and were regularly visited by the same staff to create a relationship of empathy. We were lucky to be able to follow all patients for 5 years. We report data collected at 36, 48 and 60-month observation times (Additional file 1).

Follow-up visits included assessment of VA, IOP, biomicroscopic findings, number of antiglaucomatous medications, and postoperative complications. Signs of inflammation, such as cells and flare, were graded from 0 to 4 . The MBGS [27] -using recorded photographs- was used for bleb grading by a single observer (GC) at each follow-up visit. Cystic or avascular blebs were noted.

Spectral domain optical coherence tomography (SDOCT; Topcon 3DOCT-1000, Topcon Corporation, Tokyo, Japan), already performed at the previous study end point (24 month), was repeated at the 60 -month observation time for bleb evaluation by ophthalmologists masked to clinical data (CG and LLF). A bleb was identified as successful or failed based on the presence or absence of bleb wall thickening and microcystic or hyporeflective intrableb wall structures (with respect to a $\leq 17 \mathrm{mmHg}$ target IOP level $[28,29]$ ). Thereafter, we add measurements of some bleb parameters, performed with calipers using the device's built-in software in a masked fashion. Bleb wall thickness was defined as the distance between the first reflective signal from the conjunctiva to the top of the sub-Tenon fluid space. Because the bleb wall thickness may vary along the scan, we analyzed only the maximum and minimum distances [30]. 
The whole bleb height was defined as the maximum vertical length from the outer margin of the bleb wall and the highly reflective margin of the scleral surface in the cross-sectional image.

\section{Statistical analysis}

Statistical analysis of quantitative and qualitative data, including descriptive statistics, was performed for all items. The independent Student t-test and the Mann-Whitney $\mathrm{U}$ statistic test were used for parametric and nonparametric analysis, respectively. Discrete variables were analyzed using the chi square test and Fisher exact test, as needed. Intragroup parametric and non-parametric analysis were carried on by using the paired-samples Student t-test and paired Wilcoxon signed-rank test respectively. Success was evaluated on the basis of Kaplan-Meier cumulative probability (log-rank test). To assess intraobserver reproducibility and consistency, an internal quality control system was established before the study onset by using three consecutive independent interpretations of the same SD-OCT scan, together with the unweighted Cohen kappa (k) test [31]. Data were analyzed by the Epi Info software (version 6.0, Centers for Disease Control and Prevention, Atlanta, GA, USA) and IBM SPSS Software 21.0 version (SPSS, Inc., Chicago, Ill, US). All P-values were two-sided and $P$-values less than 0.05 were considered statistically significant.

\section{Results}

Patients in the two treatment groups did not significantly differ in any of the preoperative parameters. Two women with early-onset glaucoma, aged 36 and 38 years, were included, the former in the MMC group and the latter in the OLO one.

As above said, all 40 patients completed the 60-month follow-up. During the $3^{\text {rd }}$ to $4^{\text {th }}$ year of follow-up, 3 cases $(15 \%)$ in the MMC group and 2 cases (10\%) in the OLO group developed a clinically significant cataract with VA decrease in the operated eye, and therefore underwent cataract surgery with IOL implantation (Table 1).

Mean Snellen acuity and visual field test MD at the 5years end point did not differ from the baseline in both groups (Table 1$)$. The mean preoperative IOP $( \pm$ SD) was $26.5( \pm 5.2)$ in MMC eyes and $27.3( \pm 6.0)$ in OLO eyes, without significant intergroup difference. One-day postoperatively, the IOP dropped to $5.2( \pm 3.5)$ and $9.2( \pm 5.5)$ $\mathrm{mmHg}$, respectively $(P=0.009)$. No intergroup difference was present at any scheduled postoperative time interval. The postoperative IOP reduction was significant at the 24-month endpoint in both groups $(P=0.01)$. At the extended 36-month time interval, the mean IOP was $15.6( \pm 2.6)$ in the MMC group and $15.9( \pm 2.5) \mathrm{mmHg}$ in the OLO group $(P=0.706)$, while at the $48^{\text {th }}$ month the IOP values were $15.9( \pm 2.6)$ and $15.3( \pm 3.4)$ respectively $(P=0.563)$ Finally, at the $60^{\text {th }}$ month the IOP values were $15.2( \pm 3.2)$ and $15.8( \pm 2.3)$ respectively $(P=0.579)$. The endpoint percentage IOP reduction from baseline was $40.9( \pm 14.2)$ and $42.1( \pm 13.3)$, respectively $(P=0.827)$ (Table 2 and Fig. 1). The two cases of early-onset glaucoma exhibited an IOP within the low teens without medications.

In the cases who underwent cataract phacoemulsification, the mean pre-operative IOP was $16.9( \pm 2.3)$ in MMC eyes and $17.0( \pm 2.9)$ in OLO ones, whilst the endpoint IOP was $17.2( \pm 2.5)$ and $16.5( \pm 2.7)$ respectively

Table 1 Preoperative characteristics of patients who underwent trabeculectomy, Snellen acuity and MD change, and $3^{\text {rd }}$ to $4^{\text {th }}$ year follow-up cataract surgery cases

\begin{tabular}{|c|c|c|c|}
\hline & MMC group & OLO group & $P$ \\
\hline Gender (M/F), Nº & $11 / 9$ & $12 / 8$ & $1.0^{\mathrm{a}}$ \\
\hline Age, yrs. (mean $\pm S D)$ & $63.2(7.2)$ & $65.8(6.4)$ & $0.234^{b}$ \\
\hline Right/left eyes, $N^{\circ}$ & $7 / 13$ & $11 / 9$ & $0.340^{\mathrm{a}}$ \\
\hline Type of glaucoma (POAG/PEXG), N & $12 / 8$ & $13 / 7$ & $1.0^{\mathrm{a}}$ \\
\hline Preoperative IOP,mmHg (mean \pm SD) & $26.7(5.2)$ & $27.3(6.0)$ & $0.736^{b}$ \\
\hline Baseline CDVA, decimal notation & $0.8( \pm 0.33)$ & $0.75( \pm 0.31)$ & 0.624 \\
\hline Endpoint CDVA, decimal notation & $0.8( \pm 0.40)$ & $0.8( \pm 0.35)$ & 1.0 \\
\hline Baseline MD,dB (mean $\pm S D$ ) & $-7.80(4.57)$ & $-7.41(5.35)$ & $0.805^{b}$ \\
\hline Endpoint MD,dB (mean \pm SD) & $-7.60(4.3)$ & $-7.50(5.6)$ & $0.949^{b}$ \\
\hline Preoperative medications, $\mathrm{N}^{\circ}$ (mean $\pm \mathrm{SD}$ ) & $2.5(0.3)$ & $2.6(0.2)$ & $0.222^{b}$ \\
\hline Duration of preoperative antiglaucoma therapy,yrs. (mean \pm SD) & $5.7(1.8)$ & $6.3(1.4)$ & $0.246^{\mathrm{b}}$ \\
\hline Cataract surgery cases & $3(15 \%)$ & $2(10 \%)$ & 1.0 \\
\hline
\end{tabular}

${ }^{a}$ Chi square test or Fisher exact test, as needed; ${ }^{b}$ independent Student $\mathrm{t}$ test;

$\mathrm{MMC}=$ Mitomycin-C; OLO = Ologen; SD = standard deviation;

POAG = primary open angle glaucoma; $P E X G=$ pseudoexfoliation glaucoma; $C D V A=$ Corrected Distance Visual Acuity; $M D=$ Humphrey Visual Field Analyzer Mean deviation. 
Table 2 Postoperative IOP ( $\mathrm{mmHg}$ ) in the surgical groups. Mean ( \pm SD; 95\%Cl); \% change in IOP from baseline

\begin{tabular}{llll}
\hline & MMC group & OLO group & $P^{\text {a }}$ \\
\hline $3^{\text {rd }}$ month & $14.7(3.9 ; 12.9-16.4)$ & $15.0(3.8 ; 13.3-16.7)$ & 0.806 \\
& $44.5 \%$ & $45.1 \%$ & \\
$6^{\text {th }}$ month & $14.7(4.3 ; 12.7-16.6)$ & $14.1(3.1 ; 12.6-15.4)$ & 0.615 \\
& $44.5 \%$ & $48.4 \%$ & \\
$12^{\text {th }}$ month & $15.0(3.0 ; 13.6-16.4)$ & $15.2(2.8 ; 13.8-16.4)$ & 0.828 \\
& $43.4 \%$ & $44.3 \%$ & \\
$24^{\text {th }}$ month & $16.0(2.9 ; 14.6-17.4)$ & $16.5(2.1 ; 15.5-17.4)$ & 0.536 \\
& $39.6 \%$ & $39.5 \%$ & \\
$36^{\text {th }}$ month & $15.6(2.6 ; 14.3-16.9)$ & $15.9(2.5 ; 14.6-17.2)$ & 0.706 \\
& $39.8 \%$ & $40.2 \%$ & 0.563 \\
$48^{\text {th }}$ month & $15.9(2.6 ; 14.5-17.2)$ & $15.3(3.4 ; 13.4-17.1)$ & \\
& $38.8 \%$ & $42.3 \%$ & 0.579 \\
$60^{\text {th }}$ month & $15.2(3.2 ; 13.4-16.9)$ & $15.8( \pm 2.3 ; 14.4-17.1)$ & \\
& $41.0 \%$ & $42.1 \%$ &
\end{tabular}

a Independent Student $\mathrm{t}$ test; $\mathrm{MMC}=$ Mitomycin $-\mathrm{C} ; \mathrm{OLO}=$ Ologen; $\mathrm{SD}=$ standard deviation

( $P=0.885$, and $P=0.875$, respectively). The number of antiglaucoma medications, $0.7(0.6)$ in $\mathrm{MMC}$ and 0.5 (0.7) in OLO cases did no change after cataract surgery. All cataract patients experienced a post-operative VA increase of at least 2 lines.

Table 3 reports the success rates in the study groups. At the 60-month endpoint follow-up, the values regarding complete success at $\leq 21 \mathrm{mmHg}$ target IOP were $65 \%$ and $70 \%$ respectively for the MMC and the OLO group, with a qualified success of over $85 \%$ in both. At the $\leq 17 \mathrm{~mm} \mathrm{Hg}$ target IOP, complete success percentages were $60 \%$ and $55 \%$, and qualified ones $70 \%$ and $75 \%$ respectively. At the $\leq 15 \mathrm{~mm} \mathrm{Hg}$ target IOP level complete success was recorded in $35 \%$ and $45 \%$ of cases, and qualified one in $40 \%$ and $50 \%$ of patients respectively with no significant difference at any followup time.

At 24-month follow-up, the Kaplan-Meier cumulative survival curves relating either the $\leq 21, \leq 17$, or $\leq 15 \mathrm{mmHg}$ target IOP had not showed significant intergroup differences for complete or qualified success rates. Figure 2, indicates the same parameter behavior relating complete success rates at $\leq 15 \mathrm{~mm} \mathrm{Hg}$ target IOP up to the $60^{\text {th }}$ month end point, with no significant intergroup difference (log-rank $P=0.595)$.

The area, height and vascularity MBGS scores did not generally differ on an intragroup and intergroup basis, maintaining stability till the $24^{\text {th }}$ month. One exception was height, whose mean score was higher in OLO group at the third month $(2.0 \pm 0.8$ vs $1.3 \pm 0.7 ; P=0.009$; Mann-Whitney $U$ statistic test), maintaining a higher yet not significant value till the first 24th month end point.

No significant difference was found between the 24month and the 60-month values, either with respect to central area $(P=0.729$ in MMC and $P=0.231$ in OLO group), maximal area $(P=0.769$ and $P=0.395$, respectively) and height $(P=0.408$ and $P=0.478$, respectively). The 60-month mean MBGS score values in MMC vs OLO group were $2.7 \pm 0.7$ vs $2.3 \pm 0.9(P=0.191)$ relating

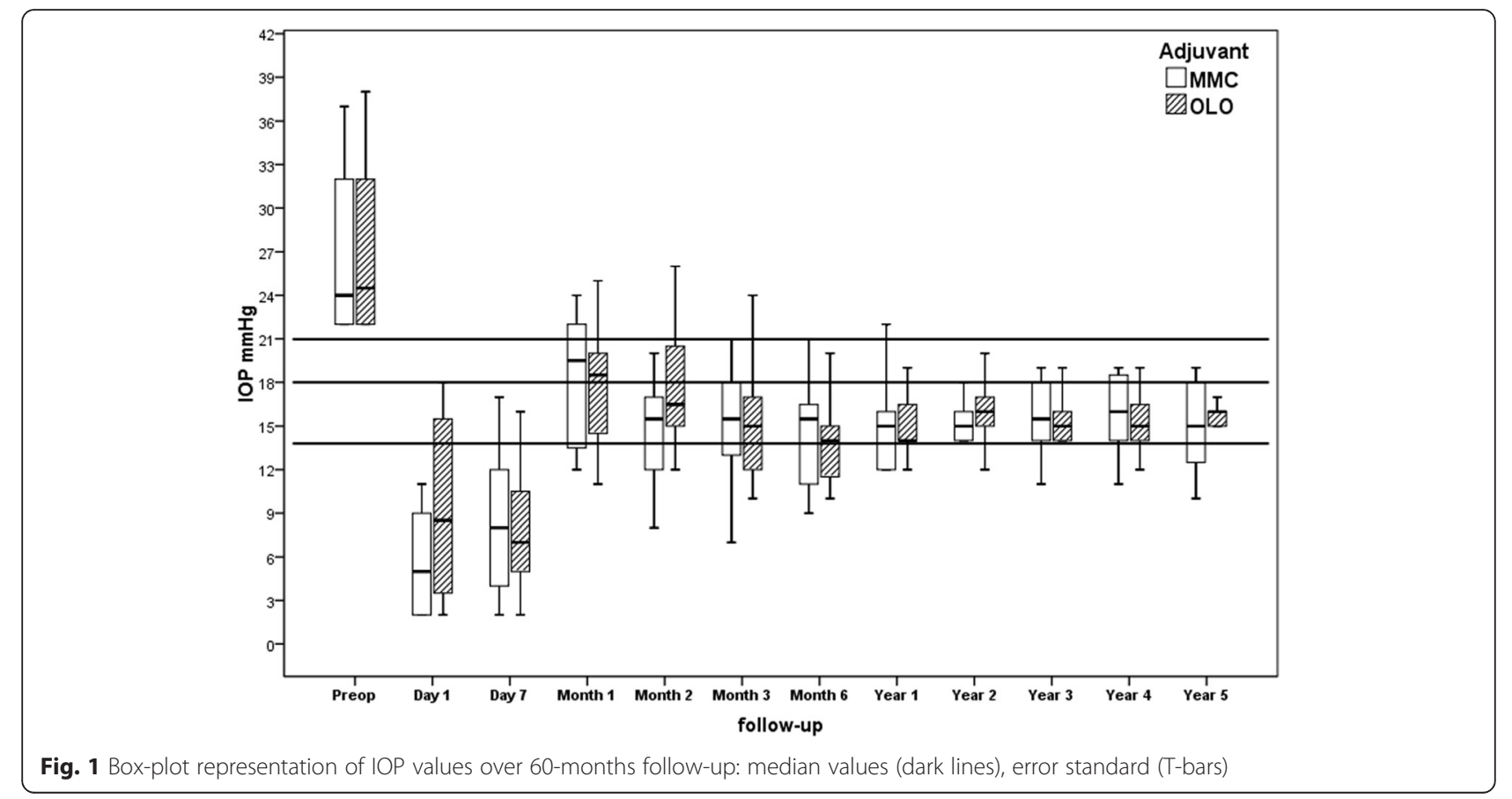


Table 3 Success rates (\%) at the 60-month follow-up study endpoint in the surgical groups at three target IOP levels

\begin{tabular}{lccc}
\hline & MMC group & OLO group & $P^{\text {a }}$ \\
\hline$\leq 21 \mathrm{mmHg}$ & & & \\
Complete success & $13(65 \%)$ & $14(70 \%)$ & 1.0 \\
Qualified success & $17(85 \%)$ & $18(90 \%)$ & 1.0 \\
$\leq 17 \mathrm{mmHg}$ & & & \\
Complete success & $12(60 \%)$ & $11(55 \%)$ & 1.0 \\
Qualified success & $14(70 \%)$ & $15(75 \%)$ & 1.0 \\
$\leq 15$ mmHg & & & \\
Complete success & $7(35 \%)$ & $9(45 \%)$ & 0.747 \\
Qualified success & $8(40 \%)$ & $10(50 \%)$ & 0.751 \\
\hline
\end{tabular}

${ }^{\mathrm{a}}$ Fisher exact test; $\mathrm{MMC}=$ Mitomycin- $\mathrm{C} ; \mathrm{OLO}=$ Ologen

central area, $2.9 \pm 0.8$ vs $2.6 \pm 1.0(P=0.378)$ relating maximal area, and $1.2 \pm 0.5$ vs $1.2 \pm 0.8(P=0.722)$ relating height, without intergroup difference. At the same time interval, central, peripheral, and non-bleb vascularity mean score values in MMC vs OLO group were $0.6 \pm 0.3$ vs $0.9 \pm 0.5,1.3 \pm 0.3$ vs $1.1 \pm 0.3$, and $1.6 \pm 1.0$ vs $1.4 \pm 1.0$, with intergroup difference $(P=0.027 ; P=0.041 ; P=0.531)$, respectively, relating the central and peripheral score. Figure 3 shows two cases with diffuse bleb, one from MMC and the other from OLO group, with a central avascular/cystic area in the former and almost normal vascularity in the latter.

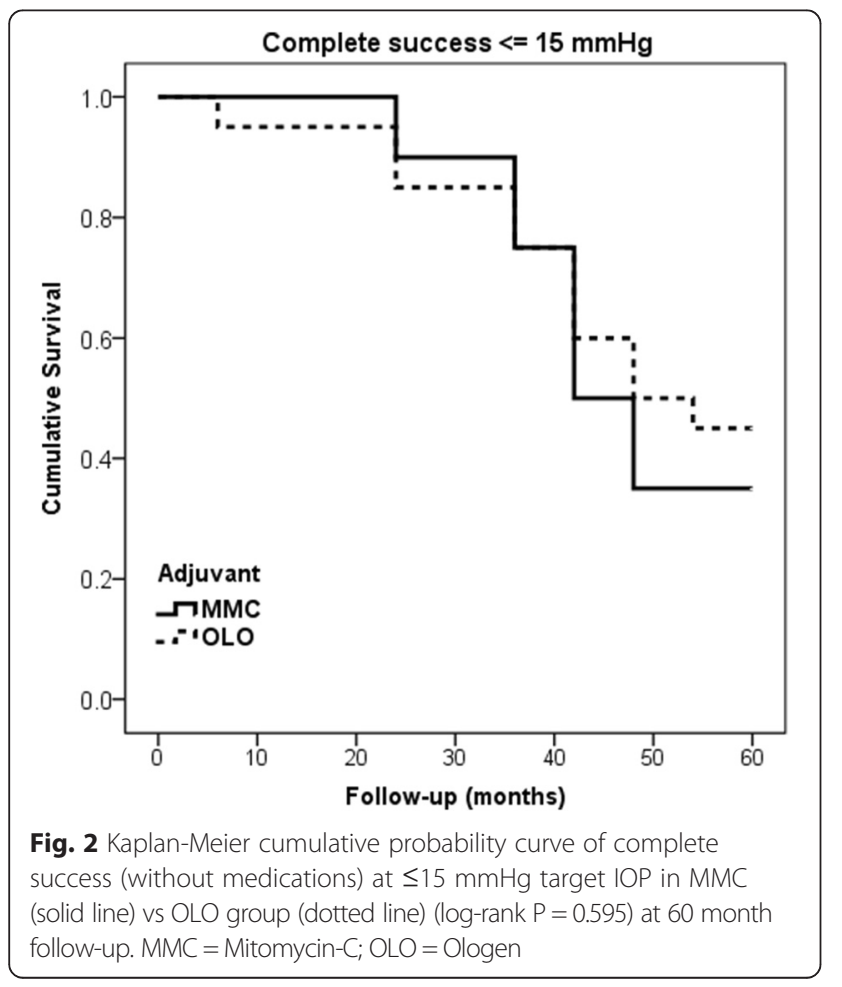

There was high intraobserver reproducibility for SDOCT analysis $(\mathrm{k}=0.7403,95 \% \mathrm{CI}$ : 0.70-0.86). Table 4 reports the successful bleb frequencies, with respect to a $\leq 17 \mathrm{mmHg}$ target IOP level, at 5-year follow-up.

Figure 4 displays examples of bleb thickness parameters obtained using the SD-OCT built-in software at the 60 -month end point. Eight eyes, 6 in the MMC and 2 in the OLO group, with a cystic thin bleb, exhibited minimum bleb wall thickness mean values significantly smaller $(54.5 \pm 14.9 \mu)$ than the remaining eyes $(134.2 \pm$ $17.3 \mu)(P=0.004)$, in agreement with the clinical appearance. No differences were found in terms of whole bleb height between groups $(140.5 \pm 20.3 \mu$ vs $129.2 \pm$ $19.3 \mu$ respectively; $P=0.079$ ).

The mean number of antiglaucoma medications was significantly reduced at end point in both groups $(P<0.0005$ in both cases): from $2.5( \pm 0.3)$ to $1.2( \pm 0.4)$ and from 2.6 $( \pm 0.2)$ to $1.4( \pm 0.3)$ in the MMC and OLO groups, respectively, without significant intergroup differences $(P=0.08)$.

The Carlo Traverso maneuver was employed in two patients in each group between the 1st and the 14th postoperative day. Four cases $(20 \%)$ in the MMC group and three cases (15\%) in the OLO group -without intergroup difference- underwent Laser suture lysis between the first and the second postoperative week. Bleb needling was performed from one to four times in seven $(35 \%)$ and six $(30 \%)$ patients respectively, again without intergroup difference.

The frequency of early postoperative complication did not significantly differ between the two groups, even if a tendency toward more frequent early bleb leakage was noted in the OLO group, and toward more frequent early hypotony, defined as an intraocular pressure (IOP) equal to or less than $6 \mathrm{mmHg}$ at the first postoperative day, and choroidal detachment in MMC one. No adverse reaction to the Ologen implant, matrix extrusion, or conjunctival erosion was present in OLO group. At the 60-month endpoint, three more cases of clinically significant cataract with VA decrease, 2 in the MMC and 1 in the OLO group, were found, to a total of 5 and 3 cases respectively during the whole follow-up. Five ( $25 \%)$ patients in the MMC group and $4(20 \%)$ in the OLO one experienced $>1$ line VA loss due to cataract or to age-related macular degeneration (AMD). Six (30 \%) cystic thin avascular blebs without oozing, as above said, were recorded in the MMC group and $2(10 \%)$ in the OLO group, without intergroup difference $(P=0.235)$ (Table 5).

\section{Discussion}

Both early and long-term complications are still reported in MMC-augmented trabeculectomy: As pointed out in our previous study [12], factors such as flaps fashioning, suturing technique and prolonged fibroblast inhibition 

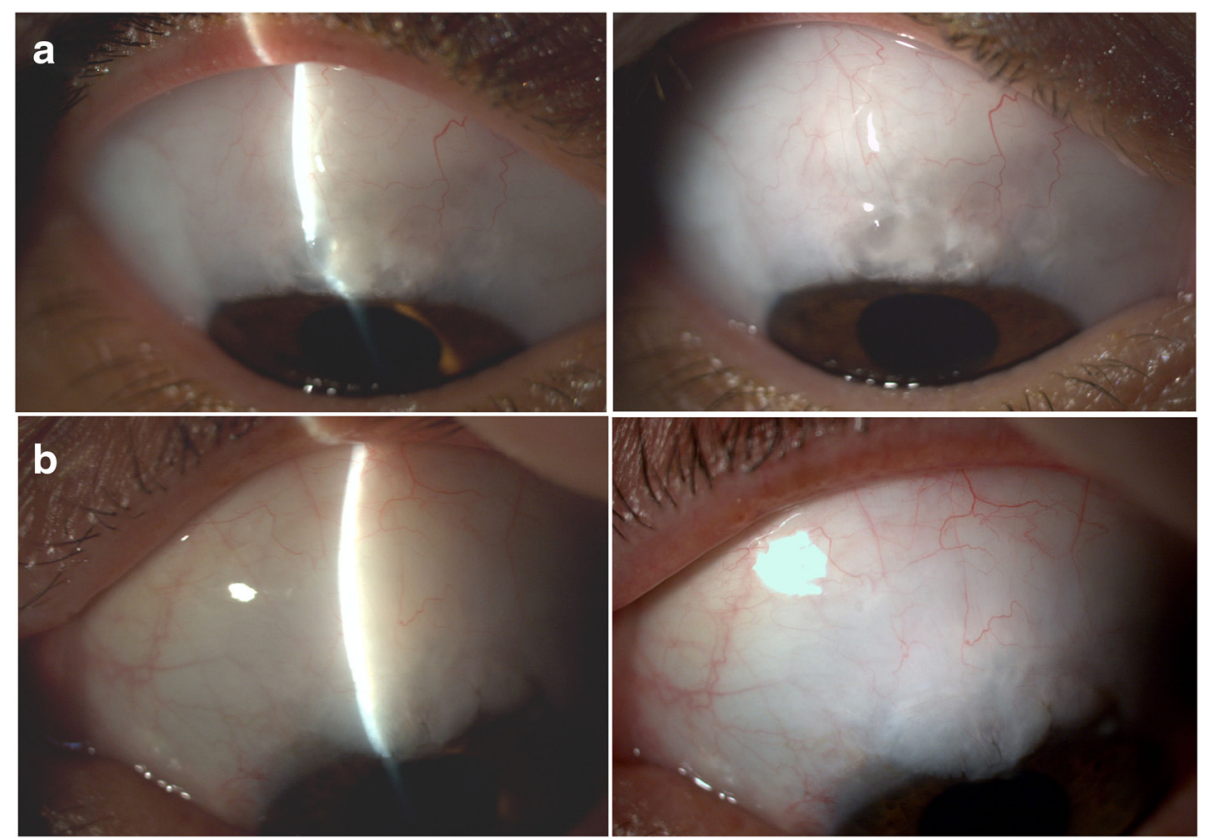

Fig. 3 Slit light and diffuser photographs of two cases. Patient a (top): 38 yrs-old woman from MMC group. a diffuse bleb with central avascular/ cystic area can be seen. Patient b (bottom): 52 yrs-old man from OLO group. A diffuse bleb with almost normal vascularity is present

-with thin bleb leaking- are responsible for these problems $[8,9,22,23,32]$.

In our previous study, the early postoperative hypotony rate is really quite high in both groups, probably due to the loose stitches without fashioning releasable sutures: the latter technique could avoid this complication [12]. Anyway, the reduced tendency to first postoperative day's hypotony in the OLO cases, when compared with the MMC ones, can be explained as a mechanical Ologeninduced aqueous outflow modulation.

The extended 5-year results indicate that the postoperative IOP behavior is quite similar in both groups, with a highly significant and stable IOP reduction, stable VA and $\mathrm{MD}$, and reduced administration of antiglaucoma medications throughout the 60-month follow-up, without intergroup differences. The equivalence in efficacy of

Table 4 60th month bleb success rates(\%) at $\leq 17 \mathrm{mmHg}$ target $\mathrm{IOP}$ in the surgical groups according to the SD-OCT analysis

\begin{tabular}{llll}
\hline & $\begin{array}{l}\text { MMC } \\
\text { group60th } \\
\text { mo }\end{array}$ & $\begin{array}{l}\text { OLO } \\
\text { group60th } \\
\text { mo }\end{array}$ & $\begin{array}{l}\text { P60th } \\
\text { mo }\end{array}$ \\
\hline $\begin{array}{l}\text { Successful bleb / eyes with } \\
\text { complete success }\end{array}$ & $10 / 12$ & $9 / 11$ & $1.0^{\text {a }}$ \\
$\begin{array}{l}\text { Failed bleb / eyes without } \\
\text { complete success }\end{array}$ & $6 / 8$ & $7 / 9$ & $1.0^{\text {a }}$ \\
$\begin{array}{l}\text { SD-OCT sensitivity } \\
\text { SD-OCT specificity }\end{array}$ & $83 \%$ & $82 \%$ & $0.627^{\text {b }}$ \\
\hline
\end{tabular}

${ }^{\mathrm{a}}$ Fisher exact test; ${ }^{\mathrm{b}} \mathrm{Chi}$-square test for the comparison of two proportions (from independent samples), expressed as a percentage;

$\mathrm{MMC}=$ Mitomycin $-\mathrm{C} ; \mathrm{OLO}=$ Ologen
OLO vs MMC is further confirmed by their endpoint success rates at the three different target IOP levels. Even if it is well known that phacoemulsification leads to an increased risk of bleb failure of approximately $33 \%$, with changes in bleb morphology and elevation in IOP of 2-3 $\mathrm{mmHg}$ [33], in our cases the temporal access phaco did not show any effect until the endpoint. The exiguous number of cases could explain this finding.

The sample size power was calculated for the IOP endpoint. Therefore, no conclusions can be drawn from the secondary endpoints, e.g. bleb morphology, number of postoperative medications and frequency of complications. We judge anyway that our results imply some interesting observations. Testifying to the persistence of the implant, the bleb height score was higher in the OLO than in MMC group at the 3rd month. Indeed, the residual implant volume added to the fluid-filled bleb spaces could justify this finding (since its biodegradation -according to the manufacturer- can last a 6-month period as above said). It is possible that a larger sample could have confirmed this difference in height for a longer period.

In studies using either morphologic grading scale evaluation or experimental 3D anterior segment SDOCT, bleb height is one of the parameters correlated with a lower IOP [28, 34-36]. Whether at 24-months or 60-months follow-up, our sample shows that the outer bleb morphology by SD-OCT was quite similar between groups. These findings imply that outer layers of a functioning bleb are not modified by the OLO implant on a 

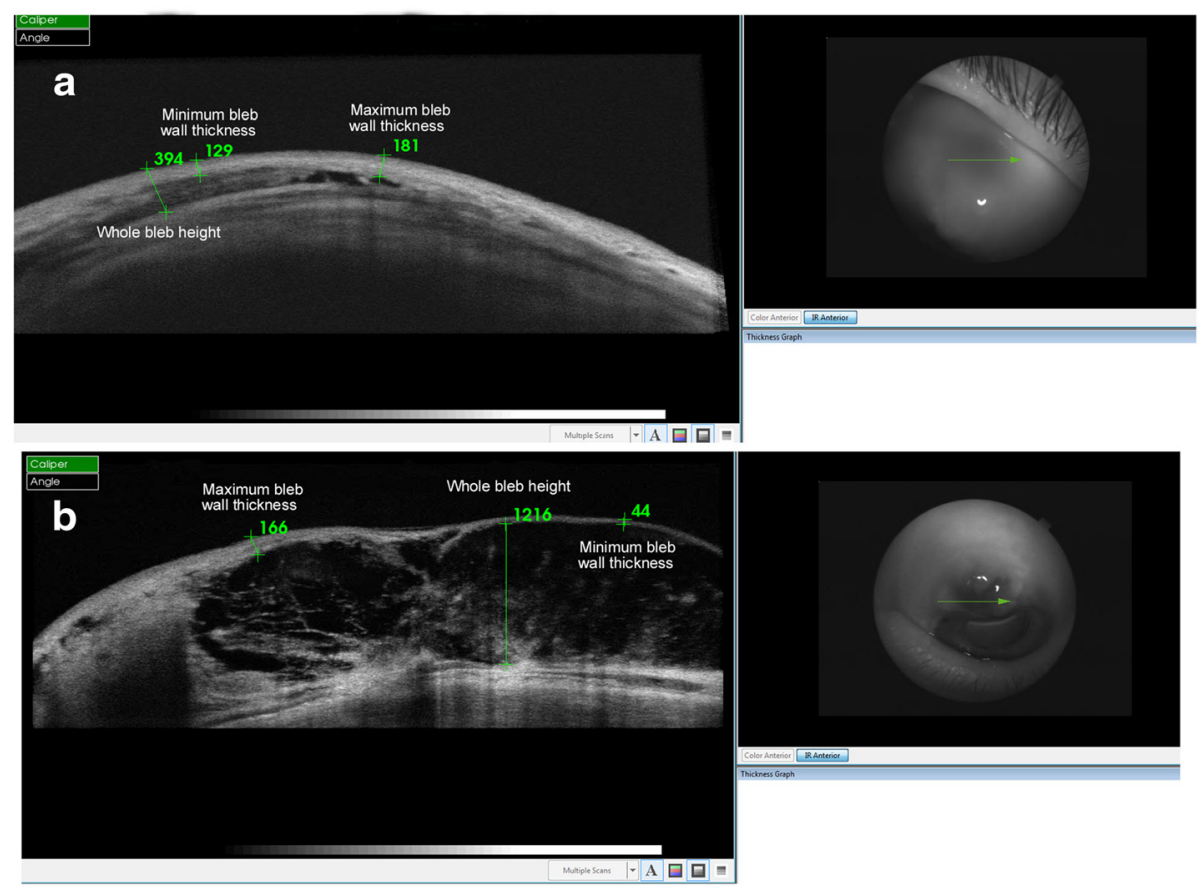

Fig. 4 SD-OCT imaging of blebs at the 60-month end point with complete success based on $\leq 15 \mathrm{mmHg}$ target IOP. (a): Thick bleb from OLO group. (b): Thin bleb from MMC group. The bleb (a), from the OLO group, exhibited a more diffuse pattern

long term basis. In summary, the OCT bleb data indicate that bleb morphology is disappointingly not strongly correlated with function. A larger sample size could perhaps reveal that the SD-OCT pattern of a successful bleb, represents a prognostic factor for longerterm success. The end point bleb wall SD-OCT analysis confirms the critically reduced thickness of cystic thin blebs in both groups. The similarity of the whole bleb height in both groups, as measured by SD OCT, is in agreement with the above said clinical judgment by MBGS criteria.

The two groups did not differ with respect to adjunctive postoperative procedures. This could once more confirm similarity between the adjuvants.

Similar rates of early complications in the two groups further corroborate the equivalence of the effect of the two adjuvants during this postoperative period. The above said tendency to 1st day greater hypotony in MMC eyes did not reach significance in our sample. The

Table 5 Frequency (\%) of 60-month postoperative complications in the surgical groups

\begin{tabular}{llll}
\hline & MMC group & OLO group & $P^{a}$ \\
\hline Cataract & $5(25 \%)$ & $3(15 \%)$ & 0.694 \\
Age-related macular degeneration & $2(10 \%)$ & $2(10 \%)$ & 1.0 \\
Loss > 1 line VA & $5(25 \%)$ & $4(20 \%)$ & 1.0 \\
Thin avascular bleb & $6(30 \%)$ & $2(10 \%)$ & 0.235 \\
\hline${ }^{a}$ Fisher exact test; MMC = Mitomycin-C; OLO = Ologen & &
\end{tabular}

${ }^{\mathrm{a}}$ Fisher exact test; $\mathrm{MMC}=$ Mitomycin- $\mathrm{C} ; \mathrm{OLO}=$ Ologen low complication rate with low-dosage MMC within 2 years is in agreement with our previous studies [24, 37-39]. The cataract and AMD incidence could be ascribed both to aging and to glaucoma surgery in our cases. As previously noted, late thin avascular blebs are relatively common with MMC use. In our sample, after 5 years MMC cases exhibit a reduced vascularity together with a trend toward more frequent thin blebs, confirming the long-term effect from fibroblast mitosis inhibition [40]. It is surprising that, besides vascularity, in our sample there is no difference in end point bleb morphology of MMC cases when compared to the OLO ones, especially taking into account the diffuse area of application of MMC in the former and the limited surface of the $6 \times 2 \mathrm{~mm}$ Ologen implant in the latter (see Fig. 3). The Ologen volume could hypothetically lift the surrounding conjunctiva from the scleral bed for a while, allowing a diffuse bleb, and/or, our sample size could imply some biased results, as above stated.

Earlier short to medium term retrospective or prospective randomized clinical trials denied our good results with Ologen. In fact, drawbacks such as lower bleb height and higher vascularity in OLO group, with a lower success rate, or higher complication rate of the Ologen-augmented trabeculectomy with respect to the simple one, or lower complete success rate even though a lower bleb-associated complication rate in Ologen vs MMC groups, are reported [10, 11, 41]. Variations in surgical procedure could justify these different outcomes. For instance, a non running suture with only two 
10-0 nylon at the flap extremities likely lead to a higher rate of positive Seidel test with flat anterior chamber. Also, it could have reduced the role of the implant in bleb development in one study [10]. Additionally, the same study included 1-month topical steroid administration, vs 2 months with variable regimen based on IPC criteria in our study. This difference could have freed the vascular reaction in OLO eyes as aforementioned, resulting in an excess of fibroblasts surrounding the implant with higher end point IOP [10]. Finally, we note that small sample sizes, techniques of application and concentration of MMC, postoperative therapeutic regimen as well as variations in composition and different manufacturers of Ologen implants over the years could also be important factors to take into consideration $[11,12,19,41]$. On the other hand, the difference between our results and those obtained by other centers in Germany, Singapore and India, could derive from the long-time known racial difference in the response to trabeculectomy, with white Caucasian performing better than Hispanic, East-Asian or Black population. The fibroblasts response to the Ologen implant could well follow this behavior [42, 43].

A one-year prospective interventional multicenter study including 30 eyes undergone trabeculectomy with MMC $(0.2 \mathrm{mg} / \mathrm{mL}, 0.1 \mathrm{~mL})$-soaked Ologen, concludes that the procedure does not seem to exert any synergistic effect in terms of IOP reduction [20]. We note that, besides the lack of a control group, the MMC soaked Ologen can be by itself the cause of "ring of steel" bleb, due to the prolonged MMC localized effect with excessive surrounding fibroblast proliferation [23].

Conversely, the results of our study are in agreement, relating efficacy and safety, with recent shorter term studies comparing Ologen-augmented vs MMC-augmented trabeculectomy [14-18].

In particular, Johnson et al [17] in a 12-month retrospective review find that Ologen allows for similar success rates as MMC comparing respectively 49 vs 50 eyes of mainly POAG patients undergoing a filtering procedure using an Ex-PRESS (Alcon, Fort Worth, TX) mini glaucoma device.

A recent systematic review and meta-analysis indicates that trabeculectomy with Ologen is a safe and effective procedure in patients with glaucoma, and is comparable with MMC in IOP-lowering efficacy, even if does not seem to offer any significant advantages compared with trabeculectomy plus MMC [13].

Under this respect, especially considering the additional cost to the Ologen implant when compared to $\mathrm{MMC}$, much larger trials are required to confirm possible advantages in terms of reduced incidence of early hypotony and late avascular-thin blebs. Once these requirements are met, the Ologen could be recommended as an alternative to MMC, at least in those cases where a target IOP in low teens is unnecessary. In the latter instance, an association between both adjuvants could be interesting.

\section{Conclusions}

Our long-term study confirms that the OLO implant demonstrates efficacy in terms of IOP reduction, with a success rate quite similar to $\mathrm{MMC}$, and at least equivalent safety. Moreover, outpatient surgery could benefit from this adjuvant if the lack of early significant hypotony will be confirmed by larger trials. Limits related to the small sample size must be overcome by further larger randomized trials to confirm the efficacy and safety of this device, and to accurately estimate any reduced risk for avascular and thin blebs with respect to MMC.

\section{Additional file}

Additional file 1: Consort flow chart. (DOCX $48 \mathrm{~kb})$

\section{Abbreviations}

OLO: Ologen ${ }^{\oplus}$; MMC: mitomycin-C; VA: visual acuity; MD: Mean deviation; MBGS: Moorfields Bleb Grading System; SD-OCT: spectral domain optic coherence tomography; IOP: intraocular pressure; POAG: primary open angle glaucoma; PEXG: pseudoexfoliative glaucoma; IPC: intensified postoperative care; IOL: intraocular lens; SD: standard deviation; AMD: age-related macular degeneration.

\section{Competing interests}

The authors declare that they have no competing interests.

\section{Authors' contributions}

SC conceived of the study and participated in its design and coordination. AC participated in the design of the study and performed the statistical analysis. FDP participated in the design and drafted the manuscript. CG and LLF participated in the acquisition of data, and helped to draft the manuscript. GC participated in the design of the study and helped to draft the manuscript. All authors read and approved the final manuscript.

Acknowledgements

No funding was obtained by any of the authors for this study.

\section{Author details}

${ }^{1}$ Department of Experimental Biomedicine and Clinical Neuroscience, Ophthalmology Section, University of Palermo (Italy), via Liborio Giuffrè, 13, 90127 Palermo, Italy. ${ }^{2}$ Department of Sciences for Health Promotion and Mother-Child Care "G. D'Alessandro", University of Palermo, Via del Vespro 127, I, 90127 Palermo, Italy. 'Department of Surgical and Biomedical Sciences, Section of Ophthalmology, University of Perugia, Piazza Menghini 1. S. Andrea delle Fratte, 06156 Perugia, Italy.

Received: 28 July 2015 Accepted: 28 February 2016

Published online: 05 March 2016

\section{References}

1. Kitazawa Y, Kawase K, Matsushita H, Minobe M. Trabeculectomy with mitomycin: a comparative study with fluorouracil. Arch Ophthalmol. 1991;109:1693-8.

2. Skuta GL, Beeson CC, Higginbotham EJ, Lichter PR, Musch DC, Bergstrom TJ, Klein TB, Falck FY Jr. Intraoperative mitomycin vs postoperative 5-fluorouracil in high-risk glaucoma filtering surgery. Ophthalmology. 1992;99:438-44. 
3. Palmer SS. Mitomycin as adjunct chemotherapy with trabeculectomy. Ophthalmology. 1991;98:317-21.

4. Jampel HD, Friedman DS, Lubomski LH, Kempen JH, Quigley H, Congdon N, Levkovitch-Verbin H, Robinson KA, Bass EB. Effect of technique on intraocular pressure after combined cataract and glaucoma surgery. An evidence-based review. Ophthalmology. 2002;109:2215-24.

5. Wilkins $\mathrm{M}$, Indar A, Wormald R. Intra-operative mitomycin C for glaucoma surgery. Cochrane Database Syst Rev. 2005;4, CD002897.

6. Cheng JW, Xi GL, Wei RL, Cai JP, Li Y. Efficacy and tolerability of nonpenetrating glaucoma surgery augmented with mitomycin $C$ in treatment of open-angle glaucoma: a meta-analysis. Can J Ophthalmol. 2009:44:76-82.

7. Palanca-Capistrano AM, Hall J, Cantor LB, Morgan L, Hoop J, WuDunn D. Long-term outcomes of intraoperative 5 -fluorouracil vs intraoperative mitomycin C in primary trabeculectomy surgery. Ophthalmology. 2009;116:185-90.

8. Anand N, Atherley C. Deep sclerectomy augmented with mitomycin C. Eye. 2005; 19:442-50.

9. Anand N, Arora S, Clowes M. Mitomycin C augmented glaucoma surgery: evolution of filtering bleb avascularity, transconjunctival oozing, and leaks. Br J Ophthalmol. 2006:90:175-80.

10. Papaconstantinou D, Georgalas I, Karmiris E, Diagourtas A, Koutsandrea C, Ladas I, Apostolopoulos M, Georgopoulos G. Trabeculectomy with OloGen vs trabeculectomy for the treatment of glaucoma: a pilot study. Acta Ophthalmol. 2010;88:80-5.

11. Rosentreter A, Schild AM, Jordan JF, Krieglstein GK, Dietlein TS. A prospective randomised trial of trabeculectomy using mitomycin C vs an ologen implant in open angle glaucoma. Eye (Lond). 2010;24:1449-57.

12. Cillino $\mathrm{S}$, Di Pace F, Cillino G, Casuccio A. Biodegradable collagen matrix implant vs mitomycin-C as an adjuvant in trabeculectomy: a 24-month, randomized clinical trial. Eye (Lond). 2011;25:1598-606.

13. He $M$, Wang $W$, Zhang $X$, Huang $W$. Ologen implant versus mitomycin C for trabeculectomy: a systematic review and meta-analysis. PLoS One. 2014;9(1):e85782.

14. Nilforushan N, Yadegari M, Hodjat P. Comparison of the success rate of trabeculectomy with OculusGen versus trabeculectomy with mitomycin C. Iranian Journal of Ophthalmology. 2011;23:3-12.

15. Senthil S, Rao HL, Babu JG, Mandal AK, Garudadri CS. Comparison of outcomes of trabeculectomy with mitomycin C vs. ologen implant in primary glaucoma. Indian J Ophthalmol. 2013:61:338-42.

16. Marey HM, Mandour SS, Ellakwa AF. Subscleral trabeculectomy with mitomycin-C versus ologen for treatment of glaucoma. J Ocul Pharmacol Ther. 2013:29:330-34.

17. Johnson MS, Sarkisian Jr SR. Using a Collagen Matrix Implant (Ologen) Versus Mitomycin-C as a Wound Healing Modulator in Trabeculectomy With the Ex-PRESS Mini Glaucoma Device: A 12-Month Retrospective Review. J Glaucoma. 2014;23:649-52.

18. Dada T, Kusumesh R, Bali SJ, Sharma S, Sobti A, Arora V, et al. Trabeculectomy with combined use of subconjunctival collagen implant and low-dose mitomycin C. J Glaucoma. 2013;22:659-62.

19. Narayanaswamy A, Perera SA, Htoon HM, Hoh ST, Seah SK, Wong TT, Aung T. Efficacy and safety of collagen matrix implants in phacotrabeculectomy and comparison with mitomycin C augmented phacotrabeculectomy at 1 year. Clin Experiment Ophthalmol. 2013;41:552-60.

20. Min JK, Kee CW, Sohn SW, Lee HJ, Woo JM, Yim JH. Surgical outcome of mitomycinC-soaked collagen matrix implant in trabeculectomy. J Glaucoma. 2013;22:456-62

21. Musch DC, Gillespie BW, Lichter PR, Niziol LM, Janz NK, CIGTS Study Investigators. Visual field progression in the Collaborative Initial Glaucoma Treatment Study the impact of treatment and other baseline factors. Ophthalmology. 2009;116(2):200-7.

22. Stalmans I, Gillis A, Lafaut AS, Zeyen T. Safe trabeculectomy technique: longterm outcome. Br J Ophthalmol. 2006;90:44-7.

23. Dhingra S, Khaw PT. The Moorfields safer surgery system. Middle East Afr J Ophthalmol. 2009;16:112-5.

24. Cillino S, Di Pace F, Casuccio A, Lodato G. Deep sclerectomy vs punch trabeculectomy: effect of low-dosage mitomycin C. Ophthalmologica. 2005; 219:281-6.

25. Marquardt D, Lieb WE, Grehn F. Intensified postoperative care vs conventional follow-up: a retrospective long-term analysis of 177 trabeculectomies. Graefes Arch Clin Exp Ophthalmol. 2004;242:106-13.
26. Traverso CE, Greenidge KC, Spaeth GL, Wilson RP. Focal pressure: a new method to encourage filtration after trabeculectomy. Ophthalmic Surg. 1984;15:62-5.

27. Wells AP, Ashraff NN, Hall RC, Purdie G. Comparison of two clinical Bleb grading systems. Ophthalmology. 2006;113:77-83.

28. Kawana K, Kiuchi T, Yasuno Y, Oshika T. Evaluation of trabeculectomy blebs using 3-dimensional cornea and anterior segment optical coherence tomography. Ophthalmology. 2009;1 16:848-55.

29. Singh M, See JL, Aquino MC, Thean LS, Chew PT. High-definition imaging of trabeculectomy blebs using spectral domain optical coherence tomography adapted for the anterior segment. Clin Exp Ophthalmol. 2009:37:345-51.

30. Jung Kl, Lim SA, Park HY, Park CK. Visualization of blebs using anteriorsegment optical coherence tomography after glaucoma drainage implant surgery. Ophthalmology. 2013;120:978-83.

31. Brennan P. Silman A. Statistical methods for assessing observer variability in clinical measures. BMJ. 1992:304:1491-4.

32. Lockwood A, Brocchini S, Khaw PT. New developments in the pharmacological modulation of wound healing after glaucoma filtration surgery. Curr Opin Pharmacol. 2013;13:65-71.

33. Patel HY, Danesh-Meyer HV. Incidence and management of cataract after glaucoma surgery. Curr Opin Ophthalmol. 2013;24(1):15-20.

34. Picht $\mathrm{G}$, Grehn F. Classification of filtering blebs in trabeculectomy: biomicroscopy and functionality. Curr Opin Ophthalmol. 1998;9:2-8.

35. Cantor LB, Mantravadi A, WuDunn D, Swamynathan K, Cortes A. Morphologic classification of filtering blebs after glaucoma filtration surgery: the Indiana Bleb Appearance Grading Scale. J Glaucoma. 2003;12:266-71.

36. Lopes JF, Moster MR, Wilson RP, Altangerel U, Alvim HS, Tong MG, Fontanarosa J, Steinmann WC. Subconjunctival sodium hyaluronate 2.3\% in trabeculectomy: a prospective randomized clinical trial. Ophthalmology. 2006;113:756-60.

37. Cillino S, Di Pace F, Casuccio A, Calvaruso L, Morreale D, Vadalà M, Lodato G. Deep sclerectomy vs punch trabeculectomy with or without phacoemulsification: a randomized clinical trial. J Glaucoma. 2004;13:500-6.

38. Cillino S, Di Pace F, Casuccio A, Cillino G, Lodato G. Deep sclerectomy vs trabeculectomy with low-dosage mitomycin C: four-year follow-up. Ophthalmologica. 2008;222:81-7.

39. Cillino S, Zeppa L, Di Pace F, Casuccio A, Morreale D, Bocchetta F, Lodato G. E-PTFE (Gore-Tex) implant with or without low-dosage mitomycin-C as an adjuvant in penetrating glaucoma surgery: 2 year randomized clinical trial. Acta Ophthalmol. 2008;86:314-21.

40. Crowston JG, Akbar AN, Constable PH, Occleston NL, Daniels JT, Khaw PT. Antimetabolite-induced apoptosis in Tenon's capsule fibroblasts. Invest Ophthalmol Vis Sci. 1998;39(2):449-54.

41. Boey PY, Narayanaswamy A, Zheng C, Perera SA, Htoon HM, Tun TA, Seah SK, Wong TT, Aung T. Imaging of blebs after phacotrabeculectomy with Ologen collagen matrix implants. Br J Ophthalmol. 2011:95:340-4.

42. Broadway D, Grierson I, Hitchings R. Racial differences in the results of glaucoma filtration surgery: are racial differences in the conjunctival cell profile important? Br J Ophthalmol. 1994;78:466-75.

43. Husain R, Clarke JCK, Seah SKL, Khaw PT. A review of trabeculectomy in East Asian People the influence of race. Eye. 2005;19:243-52.

\section{Submit your next manuscript to BioMed Central and we will help you at every step:}

- We accept pre-submission inquiries

- Our selector tool helps you to find the most relevant journal

- We provide round the clock customer support

- Convenient online submission

- Thorough peer review

- Inclusion in PubMed and all major indexing services

- Maximum visibility for your research

Submit your manuscript at www.biomedcentral.com/submit
Biomed Central 